\title{
Delirium in neurosurgery
}

\author{
Martin N. Stienen ${ }^{1,2}$ (1)
}

Received: 20 April 2019 / Accepted: 22 April 2019 / Published online: 6 May 2019

(C) Springer-Verlag GmbH Austria, part of Springer Nature 2019

The temporary (hours-days) decline in a patient's mental baseline function is commonly referred to as delirium. Its presentations include disturbances in attention, consciousness, and cognition, encompassing hyper- and/or hypoactive forms, frequently impaired sleep-wake cycle, and perceptual (hallucinations and delusions) and emotional disorders. The overall prevalence of delirium ranges around $1-2 \%$, with a higher prevalence (about $14 \%$ ) in adults $>85$ years. High-risk populations include patients undergoing surgery $(15-53 \%)$, patients admitted to the intensive care unit (ICU; 70-87\%) or (elderly) individuals in nursing homes (up to 60\%) [1].

Taking into consideration that delirium is organically caused by definition, meaning that a physically identifiable structural, functional, or chemical alteration of the brain is required for it to appear, it is evident that neurological and neurosurgical patients present particularly vulnerable populations. Still, only few prior studies set out to determine its incidence, identify predisposing and precipitating factors specifically for neurosurgical patients, and measure its impact on patient care [4, 5].

Here, Carl Moritz Zipser and coworkers are to be commended for their efforts, prospectively screening a large cohort of $n=949$ patients in a neurosurgical department for delirium [6]. The original intention of their project extended beyond the collection of scientific data, but also aimed to improve the detection, diagnosis, and management of delirium by systematic training of the hospital staff. For 1 year, elderly patients ( $\geq 65$ years) were systematically screened — using a valid instrument (Delirium Observation Screening Scale; DOS) three times daily — and younger patients were evaluated

This article is part of the Topical Collection on Neurosurgery general

Martin N. Stienen

mnstienen@gmail.com

1 Department of Neurosurgery, Stanford University Hospital and Clinics, Stanford, CA, USA

2 Department of Neurosurgery \& Clinical Neuroscience Center, University Hospital Zurich, University of Zurich,

Zurich, Switzerland upon clinical suspicion. In patients with a DOS $\geq 3$ points (suggestive of delirium), additional instruments were applied to improve diagnostic accuracy and reduce the number of false positives (Bedside Confusion Scale, Mental Status Questionnaire, Confusion Assessment Method).

Their systematic approach allowed them to obtain a robust estimate of the rate of delirium in a relatively unselected neurosurgery patient population of a tertiary Swiss teaching hospital. The authors found that one in three hospitalized patients (32.4\%) showed symptoms consistent with delirium, and they were able to establish associations between delirium and higher age, illness, functional and neurological impairment, as well as structural comorbidities of the brain (incl. dementia, degenerative disorders, epilepsies, ischemia, hydrocephalus, both subarachnoid and intracranial hemorrhage in general, as well as cerebral injury). Many of those associations are well appreciated in the literature, indicating that their collected data is valid.

Patients with delirium were subject to more extensive surgical treatment and required more ICU management, incl. mechanical ventilation. Here, unfortunately, the nature of the data collection did not allow for additional analysis of the time component: theoretically, mentioned factors could be both, the trigger or the result of delirium, respectively. While the clinical outcome in the current report only included in-hospital mortality (3.6 vs. $3.1 \%$ ), the data indicate an economical burden of delirium by longer hospitalization times (16.2 vs. 9.5 days, $p<0.001)$ and an increased need for postdischarge stationary rehabilitation. Again, it remains unclear whether delirium by itself represents an independent factor for those observations, or whether the factors associated with delirium (age, comorbidity, brain injury, extensive surgery) translate in longer length of stay and discharge location (confounded relationship).

Notwithstanding those limitations, the work adds substantially to our current understanding of delirium in neurosurgery. The author's analysis of predisposing factors highlights features that increase a neurosurgical patient's susceptibility to delirium. These are useful to identify patients-at-risk already at admission. Unfortunately, all of these factors are given, 
patient-intrinsic and unmodifiable (e.g., age and comorbidity), resulting in the inability of the care provider to act preventively. However, their analysis of precipitating factors (that can trigger delirium) points out potential targets for neurosurgeons to positively influence their patient's clinical course: e.g., by the early and effective treatment of hydrocephalus, by minimizing the impact of intracranial hemorrhage (=prevent from re-bleeding or evacuate space-occupying hematomas in carefully selected patients), by reducing additional injury to the brain (=meticulous microsurgical technique), by limiting the extend of surgery (="as much as necessary, but as little as possible"), and by an early transfer from the ICU to the normal ward (=establishing a "normal" environment with frequent mobilization, reduced noise, cognitive stimulation with calendar, clocks, regular family visits, etc.). Whether those actions truly help reduce the incidence, severity, or duration of delirium requires further investigations, but it is likely that they will add to established prevention and treatment regimes.

In a high-risk field such as neurosurgery, where even young and otherwise healthy individuals may develop delirium due to acute brain injury and critical illness, a systematic screening, such as the one proposed by the authors, appears reasonable. Moreover, it should be ascertained that physicians, nursing, and physiotherapy/occupational therapy staff are familiar with its clinical presentation, diagnosis, and up-to-date management, as multiple downsides of delirium have been identified, including an increase in morbidity, mortality, prolongation of hospitalization, increase in rates of cognitive decline and functional deterioration, and increase in the institutionalization rate $[2,3]$.

The current report offers an occasion for individual neurosurgeons and program directors to re-evaluate, whether the detection and management of delirium is satisfactory at their home institution.

\section{References}

1. American Psychiatric Association. DSM-5 Task Force (2013) Diagnostic and statistical manual of mental disorders : DSM-5. American Psychiatric Association, Arlington

2. Ely EW, Shintani A, Truman B, Speroff T, Gordon SM, Harrell FE Jr, Inouye SK, Bernard GR, Dittus RS (2004) Delirium as a predictor of mortality in mechanically ventilated patients in the intensive care unit. JAMA 291:1753-1762

3. Inouye SK (2006) Delirium in older persons. N Engl J Med 354: $1157-1165$

4. Matano F, Mizunari T, Yamada K, Kobayashi S, Murai Y, Morita A (2017) Environmental and clinical risk factors for delirium in a neurosurgical center: a prospective study. World Neurosurg 103:424430

5. Wang J, Ji Y, Wang N, Chen W, Bao Y, Qin Q, Xiao Q, Li S (2018) Risk factors for the incidence of delirium in cerebrovascular patients in a neurosurgery intensive care unit: a prospective study. J Clin Nurs $27: 407-415$

6. Zipser CM, Deuel J, Ernst J, Schubert M, von Känel R, Böttger S (2019) The predisposing and precipitating risk factors for delirium in neurosurgery: a prospective cohort study of 949 patients. Acta Neurochir. https://doi.org/10.1007/s00701-019-03927-z

Publisher's note Springer Nature remains neutral with regard to jurisdictional claims in published maps and institutional affiliations. 